\title{
Update of the Binoth Les Houches Accord for a standard interface between Monte Carlo tools and one-loop programs
}

S. Alioli ${ }^{1}$, S. Badger ${ }^{2}$, J. Bellm ${ }^{3}$, B. Biedermann ${ }^{4}$, F. Boudjema ${ }^{5}$, G. Cullen ${ }^{6}$, A. Denner ${ }^{7}$, H. van Deurzen ${ }^{8}$, S. Dittmaier ${ }^{9}$, R. Frederix ${ }^{10}$, S. Frixione ${ }^{10,12}$, M.V. Garzelli ${ }^{11}$, S. Gieseke ${ }^{3}$, E.W.N. Glover ${ }^{16}$, N. Greiner ${ }^{8}$, G. Heinrich ${ }^{8, *}$, V. Hirschi ${ }^{12}$, S. Höche ${ }^{13}$, J. Huston ${ }^{14}$, H. Ita $^{9}$, N. Kauer ${ }^{15}$, F. Krauss ${ }^{16}$, G. Luisoni ${ }^{8}$, D. Maître ${ }^{16}$, F. Maltoni ${ }^{17}$, P. Nason ${ }^{20}$, C. Oleari ${ }^{19,20}$, R. Pittau ${ }^{18}$, S. Plätzer ${ }^{21}$, S. Pozzorini ${ }^{22}$, L. Reina $^{23}$, C. Reuschle ${ }^{3}$, T. Robens ${ }^{24}$, J. Schlenk ${ }^{8}$, M. Schönherr ${ }^{16}$, F. Siegert ${ }^{9}$, J.F. von Soden-Fraunhofen ${ }^{8}$, F. Tackmann ${ }^{21}$, F. Tramontano ${ }^{25}$, P. Uwer ${ }^{4}$, G. Salam ${ }^{10}$, P. Skands ${ }^{10}$, S. Weinzierl ${ }^{26}$, J. Winter ${ }^{8}$, V. Yundin ${ }^{2}$, G. Zanderighi ${ }^{27}$, M. Zaro ${ }^{17}$

${ }^{1}$ Lawrence Berkeley National Laboratory and University of California, Berkeley, CA 94720, USA

${ }^{2}$ The Niels Bohr Institute, University of Copenhagen, DK-2100 Copenhagen, Denmark

${ }^{3}$ Karlsruhe Institute of Technology, 76131 Karlsruhe, Germany

${ }^{4}$ Humboldt-Universität zu Berlin, Institut für Physik, D-12489 Berlin, Germany

${ }^{5}$ LAPTH, Université de Savoie and CNRS, F-74941 Annecy-le-Vieux, France

${ }^{6}$ Deutsches Elektronen-Synchrotron DESY, Platanenallee 6, 15738 Zeuthen, Germany

${ }^{7}$ Universität Würzburg, Institut für Theoretische Physik und Astrophysik, D-97074 Würzburg, Germany

${ }^{8}$ Max Planck Institute for Physics, 80805 Munich, Germany

${ }^{9}$ Albert-Ludwigs-Universität Freiburg, Physikalisches Institut, D-79104 Freiburg, Germany

${ }^{10} \mathrm{CERN} / \mathrm{PH}, \mathrm{CH}-1211$ Geneva 23, Switzerland

${ }^{11}$ University of Nova Gorica, SI 5000 Nova Gorica, Slovenia

${ }^{12}$ ITTP, EPFL, CH-1015 Lausanne, Switzerland

${ }^{13}$ SLAC, Stanford University, Stanford, CA 94309, USA

${ }^{14}$ Michigan State University, East Lansing, MI 48840, USA

${ }^{15}$ Department of Physics, Royal Holloway, University of London, Egham TW20 OEX, UK

${ }^{16}$ Institute for Particle Physics Phenomenology, University of Durham, Durham, DH1 3LE, UK

${ }^{17}$ CP3, Université Catholique de Louvain, 1348 Louvain-la-Neuve, Belgium

${ }^{18}$ Departamento de Fisica Teorica y del Cosmos CAFPE, Universidad de Granada, E-18071 Granada, Spain

${ }^{19}$ Università di Milano-Bicocca, Sezione di Milano-Bicocca, 20126 Milano, Italy

${ }^{20}$ INFN, Sezione di Milano-Bicocca, 20126 Milano, Italy

${ }^{21}$ DESY Hamburg, Germany

${ }^{22}$ University of Zurich, Institute for Theoretical Physics, $\mathrm{CH}-8057$ Zurich, Switzerland

${ }^{23}$ Florida State University, Tallahassee, FL 32306-4350, USA

${ }^{24}$ Technical University Dresden, 01062 Dresden, Germany

${ }^{25}$ Dipartimento di Scienze Fisiche, Università degli studi di Napoli “Federico II" and INFN, Sezione di Napoli, I-80125 Napoli, Italy

${ }^{26}$ Prisma cluster of Excellence, Institute for Physics, Johannes-Gutenberg-Universität Mainz, D-55099 Mainz, Germany

${ }^{27}$ Rudolf Peierls Centre for Theoretical Physics, Oxford OX13PN, UK

\section{A R T I C L E I N F O}

\section{Article history:}

Received 6 September 2013

Accepted 20 October 2013

Available online 25 October 2013

\begin{abstract}
A B S T R A C T
We present an update of the Binoth Les Houches Accord (BLHA) to standardise the interface between Monte Carlo programs and codes providing one-loop matrix elements.

(C) 2013 Elsevier B.V. All rights reserved.
\end{abstract}

\footnotetext{
* Corresponding author. Tel.: +4989 32354284 .

E-mail address: gudrun@mpp.mpg.de (G. Heinrich).
} 


\section{Introduction}

The past years have seen an enormous progress in the development of programs providing next-to-leading order (NLO) corrections for multi-particle final states. This is due to new developments concerning the calculation of one-loop amplitudes as well as important progress on the Monte Carlo side to account for real radiation at NLO. The modular structure of NLO calculations allows to share the tasks between a "One-Loop Provider (OLP)", providing the virtual corrections, and a Monte Carlo program (MC) taking care of all the parts which do not involve loops. To facilitate the cross-talk between those two engines, a standard interface has been worked out during the workshop on Physics at TeV Colliders at Les Houches in June 2009, called the "Binoth Les Houches Accord (BLHA)" [1].

Meanwhile, the use of this interface [2-16] and further developments in OLP [4,7-9,17-19] and MC [3,20-27] codes have brought up the necessity to extend it with further options. The aim of this article is to provide a public document where an update of the BLHA is proposed and conventions are defined to pass parameters, calculational schemes etc., and to return less inclusive information, such as matrix elements which are not summed over all colours and helicities.

\section{Existing features of the interface}

We do not aim at an exhaustive description of the complete framework of the original interface here, referring to [1] for more details. However, we sketch the main features any extension will build upon.

The interaction between an OLP and a MC proceeds in two phases: the pre-runtime phase where order and contract files are established, and the actual runtime phase. In the pre-runtime phase, the MC creates a file called order file containing information about the setup and the subprocesses it will need from the OLP to perform the computation. A subprocess can be either a partonic subprocess or a component thereof (e.g. a specific helicity amplitude or a colour partial amplitude). The particles are identified by specifying their particle data group (PDG) code.

A flowchart of the setup between Monte Carlo program and One Loop Provider (where the new functions defined in BLHA version 2 are included already) is shown in Fig. 1. As an example, an order file written by SHERPA for $p p \rightarrow\left(Z \rightarrow e^{+} e^{-}\right)+1$ jet, using the original standards, is given in Fig. 2.

The OLP reads the order file and checks availability for each item. Then it returns a contract file telling the MC what it can provide, and labelling the individual subprocesses. An example contract file generated by GoSAM as a response to SHERPA's order file, looks like the one in Fig. 3. From this file, one can already see where an upgrade of the interface is clearly needed: as the original interface version did not contain a standard way to pass parameters, the definition of masses and widths is marked as "Ignored by OLP" in the contract file. Certainly the parameters are passed in the actual calculation, but in a non-standardised way, as an individual agreement between the particular MC and OLP. How to define a standard for the passing of parameters is described in Section 3.1.

The first integer label after each subprocess specifies that this subprocess contains only one component (if it was composed e.g. of several helicity configurations to be evaluated separately, this first label would be an integer larger than one). The second integer acts as a label for each subprocess, used at runtime to call the individual subprocesses.

After the contract has been "signed", the communication between MC and OLP proceeds via function calls. Signing a contract means that the OLP basically copies the order file and appends an OK after each setting, separated by a "|" character. For the requested subprocesses, the OLP answer should be the integer labels described above, denoting the components and the labels of the individual subprocesses. If a setting is not supported or a subprocess is not available, the OLP should indicate this with Error instead of OK after the setting. Preferably, the message "Error" should be supplemented by a specification of the error, like unsupported flag, process not available, etc. More examples can be found in [1]. In any case, the program should not proceed if Error appears in the contract file.

If the contract file does not contain any Error statements, the communication via function calls can be started. In the original standard, there were only two functions which allowed the transfer of information between the two programs. One was the function OLP_Start (char* fname, int* ierr) which should be called by the MC when initialising the runtime phase. The character string in the first argument contains the name of the contract file. The integer in the second argument is set to 1 by the function call if the contract file is accepted. In case of failure, the second argument is different from one, and an error message of the type Error: cannot handle contract file should be issued.

The second function which was already in place, but will have a different argument list with the new standards, is the function OLP_EvalSubProcess. The parameters to be passed to the OLP_EvalSubProcess function according to the original version of the interface were (in this order):

- The integer label of the subprocess (as given in the contract file).

- An array containing the components of the momenta. The momenta are placed in a one dimensional array, where physical scattering kinematics is used, i.e. $k_{1}+k_{2}=k_{3}+\cdots+k_{m}$. For each particle, the kinematics is specified by a 5-tuple: $\left(E_{j}, k_{j}^{x}, k_{j}^{y}, k_{j}^{z}, M_{j}\right)$. Thus a full $m$-particle event is specified by an array of $5 \times m$ double precision numbers filled with the $m 5$-tuples, ordered by the particle labels.

- The renormalisation scale, $\mu_{\mathrm{R}}$, as a double precision number, or an array of scales, if different scales need to be passed.

- The strong coupling $\alpha_{\mathrm{S}}\left(\mu_{\mathrm{R}}\right)$, where $\alpha_{\mathrm{S}}\left(\mu_{\mathrm{R}}\right)=1$ can be used to indicate that the MC multiplies the returned values with the adequate coupling constants.

- The array where the computed results are returned. 


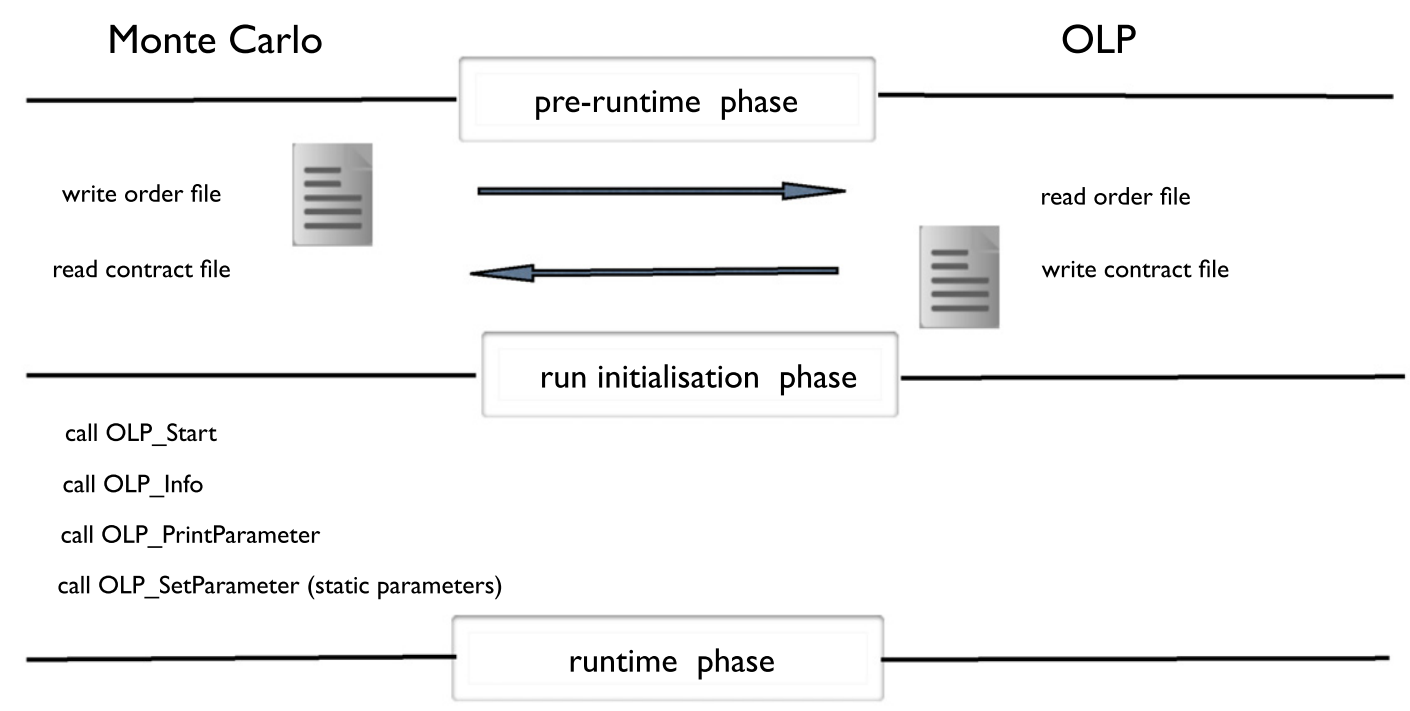

call OLP_SetParameter (dynamic parameters)

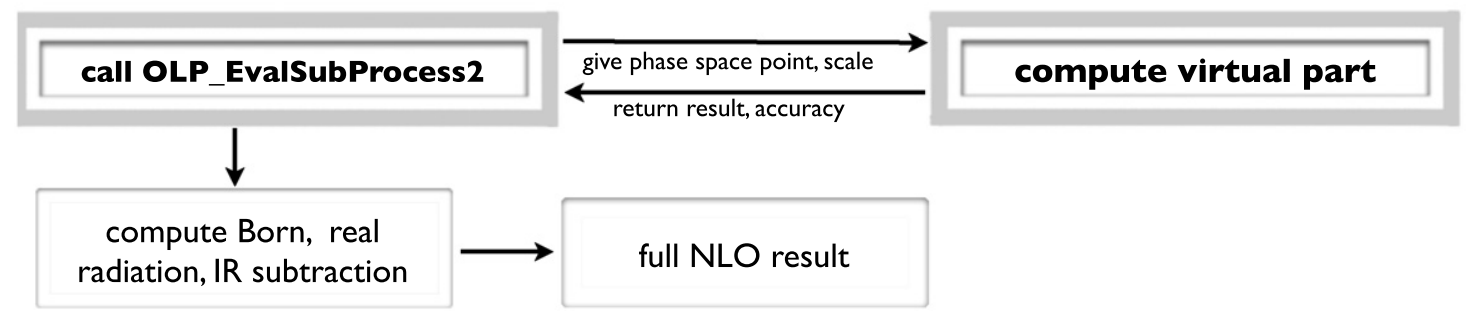

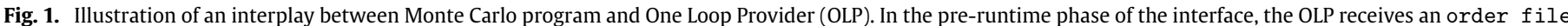

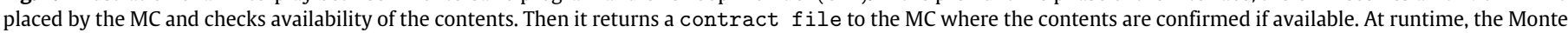

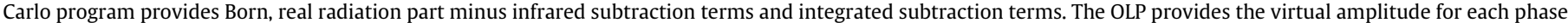
space point. The phase space integration is done by the Monte Carlo program.

\begin{tabular}{ll}
\hline \# OLE_order.lh \\
\# Created by Sherpa-1.4.1 \\
MatrixElementSquareType & CHsummed \\
CorrectionType & QCD \\
IRregularisation & DRED \\
AlphasPower & 1 \\
AlphaPower & 2 \\
OperationMode & CouplingsStrippedOff \\
& \\
Z_mass & 91.188 \\
Z_width & 2.49 \\
W_mass & 80.419 \\
W_width & 2.0476 \\
sin_th_2 & 0.221051079833 \\
& \\
\# process 1 ist & \\
$1-1->11-1121$ & \\
$211->11-11 \quad 1$ & \\
$21-1->11-11-1$ & \\
$2-2->11-1121$ & \\
$212->11-112$ & \\
$21-2->11-11-2$ & \\
\hline
\end{tabular}

Fig. 2. Example of a BLHA1 order file for the process $Z+$ jet, created by SHERPA. 


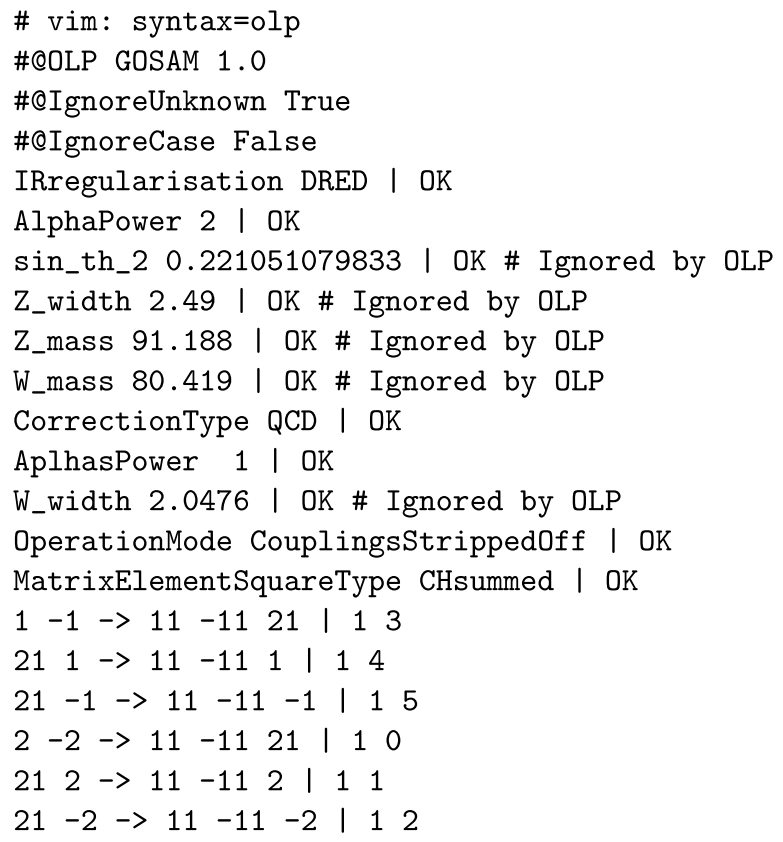

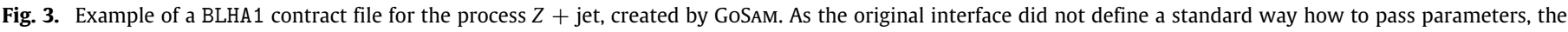
definition of masses and widths is marked as "Ignored by OLP" in the contract file, while the parameters are passed in a non-standardised way.

The returned array is expected to contain at least four real-valued double precision numbers

PoleCoeff2, PoleCoeff1, PoleCoeff0, BornSquare

which correspond to the colour- and helicity-summed (resp. averaged for the initial state) terms $A_{2}, A_{1}, A_{0}, \mid$ Born $\left.\right|^{2}$.

The conventions for the overall prefactor are as in the original proposal [1] and are briefly repeated here. The general structure of the virtual correction is given by

$$
\ell\left(\left\{k_{j}\right\}, \text { R.S., } \mu_{\mathrm{R}}^{2}, \alpha_{\mathrm{S}}\left(\mu_{\mathrm{R}}^{2}\right), \alpha, \ldots\right)=C(\epsilon)\left(\frac{A_{2}}{\epsilon^{2}}+\frac{A_{1}}{\epsilon}+A_{0}\right),
$$

where R.S. defines the infrared regularisation scheme. The Laurent coefficients $A_{j}$ are real-valued. The overall constant is given by

$$
\begin{aligned}
C(\epsilon) & =\frac{(4 \pi)^{\epsilon}}{\Gamma(1-\epsilon)}\left(\frac{\mu^{2}}{\mu_{\mathrm{R}}^{2}}\right)^{\epsilon} \\
& =(4 \pi)^{\epsilon} \frac{\Gamma(1+\epsilon) \Gamma(1-\epsilon)^{2}}{\Gamma(1-2 \epsilon)}\left(\frac{\mu^{2}}{\mu_{\mathrm{R}}^{2}}\right)^{\epsilon} .
\end{aligned}
$$

\section{New features of the interface}

The new features should serve to pass more detailed information between the MC and the OLP. However, it should always be kept in mind that the general setup is such that the MC is steering the calculation. The MC orders the virtual amplitude for the given settings, and gets back the result and a relative accuracy informing about the quality of the result. The interface does not foresee a setup where the OLP can cause changes in the MC settings.

For the pre-runtime phase, we define a number of new keywords to allow for more options in the order/contract files. The valid keywords are listed in Appendix A.

As the new standards are not backwards compatible, we propose to place the keyword InterfaceVersion, which can take the values BLHA1 or BLHA2, on top of the order file. This way, if the OLP does not support one or the other, it can issue an error message and stop without proceeding further.

Once order and contract files are established, one can distinguish two phases: The run initialisation phase and the actual runtime phase. The functions which are used for the communication between MC and OLP before exchanging phase space points and results are the following (described in more detail in the subsections below and listed in Appendix B):

- OLP_Start (char* fname, int* ierr): same as in BLHA1.

- OLP_Info(char olp_name[15], char olp_version[15], char message [255]):

the function serves to keep track of the type and version of the OLP which has been used, and to encourage proper citation. The arguments are the name of the OLP, the version, and a string which contains information about the relevant publications, for example the bibtex identifier. 
- OLP_SetParameter (char* para, double* re, double* im, int* ierr): This function is used to define static parameters at the beginning of a run, and to exchange dynamic parameters at runtime.

- OLP_PrintParameter (char* filename): prints out a list of the actual parameter settings to the file filename.

At runtime, the OLP returns the values for the virtual amplitude to the MC via the function OLP_EvalSubProcess2. As compared to the original function OLP_EvalSubProcess described in Section 2, the new function OLP_EvalSub-Process2 does not contain the passing of coupling constants anymore, as their values are now passed separately, using OLP_SetParameter. It also has a new argument appended which is useful to assess the accuracy of the result returned by the OLP. With the new argument list, the function is not backwards compatible with the original standard. Therefore, to avoid confusion with different versions, the new function is called OLP_EvalSubProcess2.

OLP_EvalSubProcess2(int* i, double* pp,double* mu, double* rval, double* acc)

The arguments are:

- $i$ : pointer to a (one element) array with the label of the subprocess as given in the contract file

- pp: pointer to an array of momenta, conventions $\left(E_{j}, k_{j}^{x}, k_{j}^{y}, k_{j}^{z}, M_{j}\right)$

- mu: pointer to the renormalisation scale

- rval: pointer to an array of return values

- acc: pointer to a one element array with the outcome of the OLP internal accuracy check (see Section 3.3).

Note: originally, the argument list of OLP_EvalSubProcess contained both $\mu_{\mathrm{R}}$ and $\alpha_{s}\left(\mu_{\mathrm{R}}\right)$. However, $\alpha_{s}\left(\mu_{\mathrm{R}}\right)$ can now be set using the new function OLP_SetParameter to pass also dynamical parameters. This setup is also clearer for mixed (e.g. QCD-EW) corrections or corrections where $\alpha_{s}$ at different scales should be used within the same calculation.

The default length of the array $\mathrm{rval}$ is four, containing the Laurent coefficients $A_{2}, A_{1}, A_{0}$ and $\mid$ Born $\left.\right|^{2}$. For the case where colour/spin correlated matrix elements are returned, the array rval must be longer. Details about the labelling conventions for such cases are given in Section 3.8 .

The last argument acc should return information about the OLP internal accuracy check(s), denoting the relative accuracy of the virtual amplitude at this phase space point as estimated by the OLP. OLPs which only provide a binary stability test will return 0 , for passed, or a large number, say $10^{5}$, for failed. More details about unstable phase space points are given in Section 3.3. The list of defined functions is given in Appendix $\mathrm{B}$, where the $\mathrm{C} / \mathrm{C}^{++}$version (with pointers in the argument list) is given. The $\mathrm{C}^{++}$version with function calls by reference as well as a Fortran (2003) module for binding with $\mathrm{C} / \mathrm{C}^{++}$can be found at http://phystev.in2p3.fr/wiki/2013:groups:sm:blha:api.

\subsection{Passing parameters}

In the first version of the interface, the standard only allowed to pass a fixed amount of information at the level of the order/contract files. However, to be able to pass also dynamical parameters like running masses, and to have more flexibility in the definition of individual parameters, we suggest the following extension.

Parameters can be passed by the function

OLP_SetParameter(char* para, double* re, double* im, int* ierr)

where the first argument is a (pointer to a) string serving as a keyword for the parameter to be set, followed by two double precision numbers so that complex parameters can also be passed (in case of real parameters, the second double is zero). The integer in the fourth argument is set by the OLP to tell the MC whether the setting of the parameter was successful.

ierr=1 means the parameter has been set successfully,

ierr $=0$ means failure: issue an error message,

ierr=2 means that the parameter is unknown or the setting is ignored (for example because it is irrelevant for the considered case), but the MC program should proceed.

The function OLP_SetParameter can be called at runtime, for every phase space point, if used to define a dynamic parameter. Obviously it can also be called once (for each particular parameter that needs to be passed) if this is a static parameter needed only at the run initialisation phase.

Further, we propose a routine OLP_PrintParameter (char* filename) giving out a list of the actual parameter settings used in the calculation, where filename is the name of the output file. The intention of this function is just to inform the user, consistency between the parameters will not be checked. The output format of OLP_PrintParameter should be

ParameterName Value State,

where the separator is a space, and Value can be complex, denoted by (real part, imaginary part) in case it is complex. State can serve to distinguish the parameters set by OLP_SetParameter or defined by Model from fixed internal ones and is optional (as this info may not be readily available in all programs).

\subsection{Defining the model}

We distinguish two alternative ways of model definition, which will be denoted by "keyword model" respectively "UFO model" in the following.

Model definitions offer the possibility to define some global settings in the order file, which are intrinsic to the model (e.g. SM, MSSM), which is used. This is done using the required keyword Model. For example, Model: SMdiag should set the CKM matrix to unity globally.

In the "keyword model" setup, the parameters that need to be set within a certain model are passed via PDG codes [28] and keywords with naming conventions as specified in Fig. 4 for the Standard Model. The numbers in parenthesis after mass and width denote the particle's PDG code. 


\begin{tabular}{|l|l|}
\hline keyword & parameter \\
\hline mass (5) & b quark mass \\
mass (6) & top quark mass \\
width (6) & top quark width \\
sw2 & $\sin ^{2} \theta_{w}$ \\
vev & SM vacuum expectation value \\
Gf & $G_{\text {Fermi }}$ \\
VV12 & $V_{u d}$ \\
$\vdots$ & \\
\hline
\end{tabular}

Fig. 4. List of keywords to define parameters to be passed by the function OLP_SetParameter.

In the "UFO model" setup, the parameters are defined in UFO (Universal Feynrules Output) [29] format, which is particularly useful for calculations beyond the Standard Model. The import of the UFO model file should be specified in the order file by

Model ufo:/path_to_ufo_model-directory/.

The UFO format also provides human readable name attributes for the model parameters, as well as the SLHA identifiers [30-32]. OLPs which support the import of UFO model files typically also support the name attributes. The UFO model setup entails the use of a SLHA parameter card to initialise the runtime phase. This requires an additional keyword ParameterCard, followed by the path to the SLHA parameter card, to be placed into the order file when using the UFO model setup. The parameters which are set by reading in the SLHA parameter card do not need to be set again by OLP_SetParameter. However, OLP_SetParameter needs to be used at runtime for the dynamic parameters. In this case the SLHA block name should appear as a prefix prepended to the parameter name, in the form $<$ BlockName $>\& \&<$ ParamName $>$. To avoid confusion, this requires that the characters '\&\&' should never appear in any block or parameter name.

Note that we do not require the capability to import UFO model files to belong to the "minimal standard". However, at least one of the two ways described above to define the model parameters should be implemented to comply with the standard.

\subsection{Treatment of unstable phase-space points}

In a complex multi-leg one-loop calculation, some of the phase space points may lead to large numerical cancellations within the virtual amplitude, leading to a result which is not trustworthy at this particular point. It is the task of the OLP to make precision tests, such that it can trigger some rescue procedure in case the phase space point is found to be problematic. In order to steer precision requirements from the order file, we introduce the following.

- The OLP should pass information about the quality of the virtual result at a given phase space point back to the MC, by means of the function OLP_EvalSubProcess2. The last argument of OLP_EvalSubProcess2 should be a (double precision) number "acc", giving the relative accuracy the OLP attributes to the finite part of the loop amplitude at this phase space point. Note that this value will depend on the type of internal stability test(s) made by the OLP. The assessment of the internal "relative accuracy" can therefore vary between different OLPs and even within the same OLP if it can switch between different types of stability tests. However, it allows the MC to give a more reliable overall accuracy estimate for the cross section than if it had just a "pass" or "fail" information from the OLP side.

- The user should be able to specify the overall accuracy he would like to reach (this is typically done in the MC runcard). For this purpose there should be an (optional) keyword in the order file which specifies the target accuracy, called AccuracyTarget, denoting a relative accuracy. The OLP can use it to decide whether an internal rescue system should be triggered if its stability test outcome gives a value larger than AccuracyTarget. If AccuracyTarget is not specified, the OLP will use its internal settings to decide if a rescue system should be triggered.

It could be that a particular OLP is not able to provide a number for the relative accuracy, but only can deliver a "binary" test outcome passed or failed. In this case, acc $=0.0$ stands for passed, and in the case of failed, acc can be set to a large value, for example $10^{5}$. In order to allow the MC to distinguish the "binary test" return value for passed from a "quantitative test" return value for passed, we can require that quantitative stability tests will return a perfect accuracy as working precision (typically 1d-17, 1d-34), while binary stability tests return exactly 0.0 .

- There should be the possibility that the OLP prints points classified as "unstable" to a file for monitoring purposes. Therefore we propose an optional keyword DebugUnstable in the order file which can be used to keep information about points classified as "unstable". In the case of DebugUnstable True, the phase space point should be printed to a file to allow further diagnostics. The format of such a debug file can be defined internally within the OLP. The threshold which defines whether a point is classified as "unstable" (after eventual rescue attempts) does not need to be equal to the value of AccuracyTarget, but this should be the default. In any case, DebugUnstable is mostly for OLP developers for monitoring purposes.

We emphasise again that after all, it is left to the MC to decide what to do with the phase space point, based on acc returned by OLP_EvalSubProcess2. 


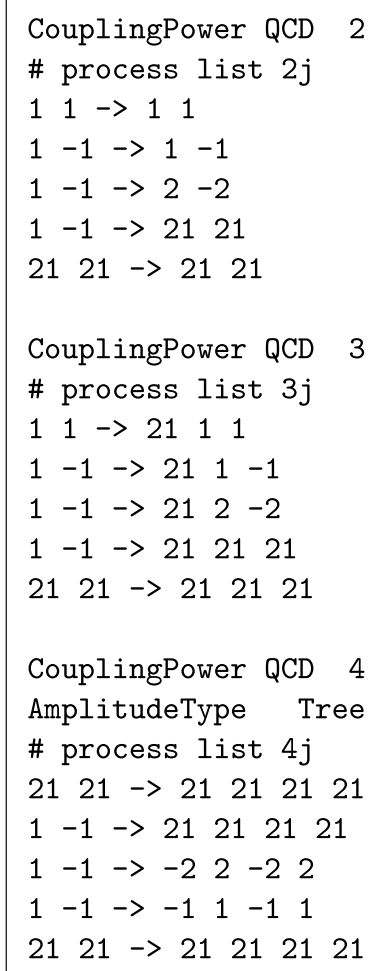

Fig. 5. Example of the part of an order file containing different settings for different sets of subprocesses.

\subsection{Different powers of coupling constants, merging different jet multiplicities}

So far, the interface was tailored to NLO calculations for a fixed jet multiplicity, and focused on QCD corrections rather than electroweak corrections. However, mixed QCD-EW corrections, or expansions in parameters other than $\alpha_{s}$ or $\alpha$, require a more flexible scheme to define the desired orders in coupling constants.

In addition, recent developments [14,33-38] propose a merging method for matched NLO predictions with varying jet multiplicity. In order to calculate merged samples, the Monte Carlo program needs to ask the OLP for one-loop matrix elements with different jet multiplicities and therefore different powers of the coupling constant.

These situations can be accounted for by allowing different settings for different subprocesses, see Fig. 5. A setting is valid until it is explicitly overwritten. This setup can be used for merged samples as well as mixed QCD/EW corrections. It can also be used to pass additional information referring only to particular subprocesses, as indicated e.g. by AmplitudeType Tree. In the latter case, the return value rval is a one element array containing the Born matrix element squared, $\mid$ Born $\left.\right|^{2}$.

\subsection{Extras}

The keyword Extra can be used to write special requirements relevant to the OLP into the order file. If there is a way to generate the order file for the OLP from the MC run card, the MC will write them to the order file, but otherwise ignore them. The difference between optional keywords and keywords preceded by the "Extra" flag is that optional keywords have defined names (as listed in Appendix A.2), while keywords flagged by "Extra" can be very OLP specific and do not have a standard name.

For example, requirements concerning a colour expansion, or passing only particular helicity configurations, could be put under the Extra flag. The Extra flag can also be used to define that MC and OLP should use the MiNLO [39] procedure, if the latter is available on both sides.

With regards to overall averaging and symmetry factors, the default is that amplitudes are summed over final state colours and polarisations and averaged over initial state colours and polarisations, and that symmetry factors for identical final state particles are included. A possibility to change this could look as follows.

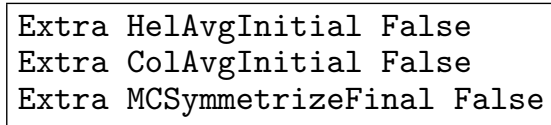

\subsection{Electroweak corrections}

In the case of electroweak (EW) corrections, it is of particular importance to check the consistency of the parameters, for example the relation between $M_{Z}, M_{W}$ and $\sin ^{2} \theta_{w}$. The scheme is set in the order file by the keyword EWScheme, which can take the values 


$\begin{array}{ll}\text { \# OLE_order.lh } \\ \text { \# Created by Sherpa-2.0.0 } \\ \text { InterfaceVersion } & \text { BLHA2 } \\ \text { Model } & \text { SMdiag } \\ \text { AmplitudeType } & \text { CHsummed } \\ \text { CorrectionType } & \text { QCD } \\ \text { IRregularisation } & \text { DRED } \\ \text { WidthScheme } & \text { ComplexMass } \\ \text { EWScheme } & \text { alphaGF } \\ \text { AccuracyTarget } & 0.0001 \\ \text { DebugUnstable } & \text { True } \\ \text { Extra } & \text { Line1 } \\ \text { Extra } & \text { Line2 } \\ \text { \# process list } & \\ \text { CouplingPower QCD } & 2 \\ \text { CouplingPower QED } & 0 \\ 1-1->6-6 & \\ -11->6-6 & \\ 2121->6-6 & \\ \text { CouplingPower QCD } & \\ \text { CouplingPower QED } & \\ 1-1->6-621 & 0 \\ 121->6-61 & \\ -11->6-621 & \\ -121->6-6-1 & \\ 211->6-61 & \\ 21-1->6-6-1 & \\ 2121->6-6 \text { 21 } & \\ & \end{array}$

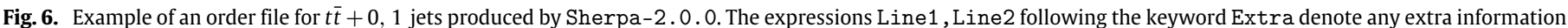
which may be relevant for the OLP.

alphaGF (also known as $G_{\mu}$-scheme), alpha0, alphaMZ, alphaRUN, alphaMSbar, OLPDefined. Then the parameters are set using OLP_SetParameter. The OLP imports these parameters. The integer in the last argument only indicates if the import was successful or not. It is left to the user to ensure consistency of the parameters within the given scheme.

\subsection{An example order file}

An example for an order file is shown in Fig. 6. The lines following the keyword Extra stand for any extra information which may be relevant to the OLP (which, in this example, is parsed from the SHERPA runcard to the order file).

\subsection{Colour and spin correlated tree amplitudes}

Going beyond the generic case of colour and helicity summed matrix elements, it becomes difficult to satisfy special needs of different programs with one global standard. Results for polarised amplitudes in general depend on phase conventions, and approximations in a colour expansion, like e.g. "leading colour", will very likely be defined differently in different codes. Nonetheless, the interface in principle allows to pass very specific information using the Extra flag. However, the details probably remain to be implemented and tested individually between specific programs before trying to establish any standards.

Below we only focus on a particular example going beyond CHsummed, which is passing colour and spin correlated tree amplitudes. The definitions are oriented at the case where they are used for the construction of NLO subtraction terms in the Catani-Seymour formalism [40]. file:

The MC can ask for colour or spin correlated tree matrix elements by writing one (or several) of the following keywords into the order

\begin{tabular}{|ll|}
\hline AmplitudeType ccTree & \# colour correlated tree amplitude \\
AmplitudeType scTree & \# spin correlated tree amplitude
\end{tabular}

The defaults for AmplitudeType are Loop and CHSummed. If only Tree or Loop are specified, this automatically means CHSummed.

The new setup to define individual settings for blocks of subprocesses can be used to call certain subprocesses twice: one for the colour/spin correlated tree case and one for the loop case. An example is given by 


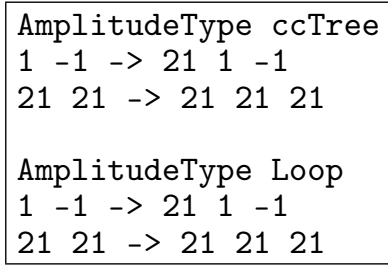

If the amplitude returned by the OLP is not colour/polarisation summed, the values to be returned by OLP_EvalSubProcess2 form a matrix in colour respectively Lorentz space. To pass the values in an unambiguous way, it is necessary to define the order in which the matrix elements are written into the array rval returned by the OLP.

\section{Colour correlations}

The colour correlated matrix elements

$$
C_{i j}=\left\langle\mathcal{M}\left|\mathbf{T}_{i} \cdot \mathbf{T}_{j}\right| \mathcal{M}\right\rangle
$$

can be defined to be real valued quantities and independent of the particular colour basis chosen. Note that $\mathbf{T}_{i} \cdot \mathbf{T}_{j}$ is symmetric under exchange of $i$ and $j$. Further, $\mathbf{T}_{i}^{2}=C_{i} \mathbf{1}$ with $C_{i}=C_{F}$ if the leg $i$ belongs to the $\mathbf{3}$ or $\overline{\mathbf{3}}$ representation of SU(3), and $C_{i}=C_{A}$ if the leg belongs to the $\mathbf{8}$ representation, and $\left\langle\mathcal{M}\left|\mathbf{T}_{i} \cdot \mathbf{T}_{i}\right| \mathcal{M}\right\rangle=C_{i}\langle\mathcal{M} \mid \mathcal{M}\rangle=C_{i}|\mathcal{M}|^{2}$ is just proportional to the tree level amplitude squared.

The minimal information that needs to be passed for colour correlated matrix elements is the upper non-diagonal $n(n-1) / 2$ elements of an $n \times n$ matrix, where $n$ is the number of legs attached to the process of interest. Entries belonging to non-coloured legs should be ignored.

For a process which is flagged as AmplitudeType ccTree the OLP should (through rval returned by OLP_EvalSubProcess2) return an array of length $n(n-1) / 2$ such that the element at position $i+j(j-1) / 2$ (counting external legs and elements in the array starting from zero) contains the result for $C_{i j}$ with $i<j$.

Here and in the following we assume that $i$ labels the emitter and $j$ the spectator.

\section{Spin correlations}

When gluons are present at the Born level, the spin correlated Born amplitude is obtained by Lorentz-contracting the corresponding Born terms with the gluon polarisation vectors. It can be shown that these amplitudes can be written in terms of

$$
S_{i j}=\left\langle\mathcal{M}_{i,-}\left|\mathbf{T}_{i} \cdot \mathbf{T}_{j}\right| \mathcal{M}_{i,+}\right\rangle,
$$

with

$$
\left\langle\mathcal{M}_{i,-}\left|\mathbf{T}_{i} \cdot \mathbf{T}_{j}\right| \mathcal{M}_{i,+}\right\rangle=\sum_{\lambda_{1}, \ldots, \lambda_{i-1}, \lambda_{i+1}, \ldots, \lambda_{n}}{ }\left\langle\mathcal{M}_{\lambda_{1}, \ldots, \lambda_{i-1},-, \lambda_{i+1}, \ldots, \lambda_{n}}\left|\mathbf{T}_{i} \cdot \mathbf{T}_{j}\right| \mathcal{M}_{\lambda_{1}, \ldots, \lambda_{i-1},+, \lambda_{i+1}, \ldots, \lambda_{n}}\right\rangle_{\mathcal{C}}
$$

Here, |\rangle$_{C}$ indicates an object which is a vector in colour space only, and we have made explicit the spin dependence by the subscripts $\lambda$ and the fixed \pm polarisation of the gluon $i$ of interest. Depending on the organisation of the subtraction terms, non-trivial colour correlations $i \neq j$ may be needed. Since $i$ is defined to be the gluon index, we have $S_{i j} \neq S_{j i}$ and $S_{i j} \in \mathbb{C}$, so in general the communication of a complex $n \times n$ matrix is required. As for the plain colour correlated matrix elements, entries corresponding to non-coloured legs or coloured legs for which the infrared singular limit does not involve spin correlations (i.e. everything but gluons for all practical purposes) should be ignored.

For a process flagged as AmplitudeType scTree the OLP should (through the standard OLP_EvalSubProcess 2 method) return an array of length $2 n^{2}$, such that the elements at positions $2 i+2 n j$ and $2 i+2 n j+1$ (counting external legs and elements in the array starting from zero) contain $\operatorname{Re}\left(S_{i j}\right)$ and $\operatorname{Im}\left(S_{i j}\right)$, respectively.

A consistent setup of spin correlations in this way further requires the MC code to obtain the gluon polarisation vector $\epsilon_{+}^{\mu}(p, q)$ as used by the OLP, given the gluon momentum $p$ and a reference vector $q$. The gluon polarisation vectors can be passed via a dedicated function

OLP_Polvec(double* p, double* q, double* eps)

where $\mathrm{p}$ and $\mathrm{q}$ are arrays of size 4, denoting the momentum $p$ of gluon $i$ and the corresponding reference vector, and eps is an array of size 8 containing the four complex components of $\epsilon_{ \pm}^{\mu}(p, q)= \pm \frac{1}{\sqrt{2}} \frac{\left\langle q^{\mp}\left|\gamma^{\mu}\right| p^{\mp}\right\rangle}{\left\langle q^{\mp} \mid p^{ \pm}\right\rangle}$, in a form where real and imaginary parts are alternating.

Returning all possible colour and spin correlations could potentially become a significant overhead; we leave it to the OLP code to support the calculation of a single correlator. Information about the correlator to be returned could be passed via the OLP_SetParameter method right before the OLP_EvalSubProcess 2 call in question.

Obviously, if one of the contractors cannot provide the detailed colour or helicity information requested, the calculation should exit at the stage of OLP_Start.

\subsection{Restrictions such as diagram filters, exploitation of special symmetries, etc.}

The keyword ExcludedParticles in the order file can be used to remove unwanted particles from the code generation. Restrictions like confining the set of diagrams to resonant diagrams only can be set in the MC input card. OLP specific restrictions can be imposed using the keyword Extra. 


\section{Conclusions}

This writeup summarises the update of the standard interface between Monte Carlo programs and one-loop matrix element providers which has been initiated at the Les Houches 2009 workshop on Physics at TeV Colliders, called the "Binoth Les Houches Accord (BLHA)". The setup meanwhile has been implemented by several groups and facilitates the automation of NLO calculations.

The experience gained meanwhile with the original setup fed into the discussion about an extension of the standards, such that the interface can be used in a wider and more flexible context. The outcome of the discussion between a large number of Monte Carlo and One-Loop Providers (OLPs) is summarised in the present document, which is intended to serve as a reference for the new standards. This should increase the flexibility of both Monte Carlo programs to import virtual corrections where available and of OLPs to team up with different Monte Carlo programs. This is an important step forward, as different MCs and OLPs have different focus and strengths concerning for example the multiplicity of final states, particle masses, electroweak corrections, BSM capability, etc. We therefore hope that BLHA version 2 will contribute to the goal of extending the comparison of LHC data with theoretical results consistently beyond the leading order.

\section{Acknowledgements}

We would like to thank the CERN TH/LPCC Institute on SM at the LHC 2012 for hospitality and for providing a stimulating environment to discuss this interface. We would also like to thank the Les Houches 2013 workshop organisers for providing a platform (e.g. Wikipages) and again a stimulating environment to work out this Accord. GH would like to dedicate this document to Thomas Binoth, and thank everybody who contributed to the creation and implementation of the Accord, both BLHA1 and BLHA2.

\section{Appendix A. List of valid keywords for order/contract file}

\section{A.1. Required keywords}

InterfaceVersion: can take the values BLHA1 or BLHA2. This clarifies already in the pre-runtime phase if the new standards are supported by both MC and OLP.

Model: SMdiag, SMnondiag, MSSM, ufo:/abs-path-to-ufo-file/. For BSM standards, the UFO [29] format is most convenient. CouplingPower QCD: integer which specifies the $\alpha_{\mathrm{S}}$ power of the Born cross section. Can be used for sub-processes as well, where it also refers to cross section rather than the amplitude level.

CorrectionType: the type of higher order correction. Standard values are QCD, QED, corresponding to expansions in $\alpha_{\mathrm{S}}, \alpha$. IRregularisation: the infrared regularisation scheme used. Possible choices for QCD are CDR, DRED.

\section{A.2. Optional keywords}

AccuracyTarget: the target accuracy the user would like to achieve. This is a relative accuracy the OLP can use to decide if a rescue system should be triggered if the result of internal stability checks returns a value which is larger than AccuracyTarget.

DebugUnstable: can take the values True or False. Indicates that the unstable phase space points should be printed to a file for further diagnostics. In order to decide what is classified as "unstable phase space point", the threshold given by AccuracyTarget or an internal OLP threshold can be used.

CouplingPower QED: integer which specifies the $\alpha$ power of the Born cross section. The default is zero. Can also be used for other couplings in the form CouplingPower $\mathrm{gX}$ if the coupling gX is defined (for example through an UFO module). Note that CouplingPower QCD needs to be defined explicitly, while CouplingPower QED will be set to the default value (zero) if not specified.

AmplitudeType: the default is Loop. It can also take the values ccTree (colour correlated tree), scTree (spin correlated tree), Tree, LoopInduced. Tree without "cc" or "sc" implies CHsummed.

Extra: can be used to write special requirements relevant to the OLP into the order file. If the MC generates the order file from a run card where such requirements are specified, it will copy them into the order file but otherwise ignore them.

ParameterCard: gives the path to the SLHA parameter card if the UFO model setup is used.

MassiveParticles: defines a list of massive particles at the level of the order file, for example MassiveParticles 5 . The separator is a space. This also implies that the light-quark masses are set to zero.

LightMassiveParticles: useful if mass regularisation instead of dimensional regularisation is used (e.g. for electroweak corrections). It defines the set of particles where only $\log (m)$ terms are kept, but not power suppressed ones.

ExcludedParticles: can be used to exclude particles which are contained by default in Model. The particles should be listed after the keyword, denoted by their PDG numbers, and separated by a space.

MassiveParticleScheme: a standard choice is OnShell.

SubdivideSubprocess: this flag defines whether a given process is represented in a split form to allow for multi-channel Monte Carlo sampling. May be useful e.g. in the case of colour expansions. Can take the values True or False (default).

EWScheme: used schemes (discussed in the text) can be flagged by the keywords alpha0, alphaMZ, alphaGF, alphaRUN, alphaMSbar, OLPDef ined (default).

WidthScheme: defines the treatment of unstable particles. Standard values are ComplexMass, FixedWidth, RunningWidth, PoleApprox. 


\section{A.3. List of keywords contained in the original proposal which will be dismissed}

MatrixElementSquareType: replaced by AmplitudeType, declared CHsummed as default. Originally this flag was intended to specify colour $(C)$ and helicity $(H)$ treatment. Possible values were defined as CHsummed, Csummed, Hsummed, NOTsummed.

The new default CHsummed also implies an average over initial state colours and polarisations. The flag Extra can be used to accommodate for the keywords HelAvgInitial, ColAvgInitial, MCSymmetrizeFinal, which can be set to False if the factors included by default should be switched off.

ModelFile: the model file from which parameters have to be read. Keyword replaced by Model.

OperationMode: the operating mode of the OLP. This optional flag was intended to specify OLP-defined conventions or approximations to the one-loop contribution. Typical operating modes are CouplingsStrippedOff, LeadingColour, HighEnergyLimit. The keyword CouplingsStrippedOff turned out to be ambiguous in the presence of EW couplings and therefore will not be used any longer.

ResonanceTreatment: has been replaced by WidthScheme, as, for example, the complex-mass scheme also affects non-resonant propagators.

\section{Appendix B. List of defined functions}

We list here the definitions of the functions needed for a working interface in the $\mathrm{C} / \mathrm{C}^{++}$version (with pointers in the argument list). A Fortran (2003) module for binding with $\mathrm{C} / \mathrm{C}^{++}$can be found at http://phystev.in2p3.fr/wiki/2013:groups:sm:blha:api.

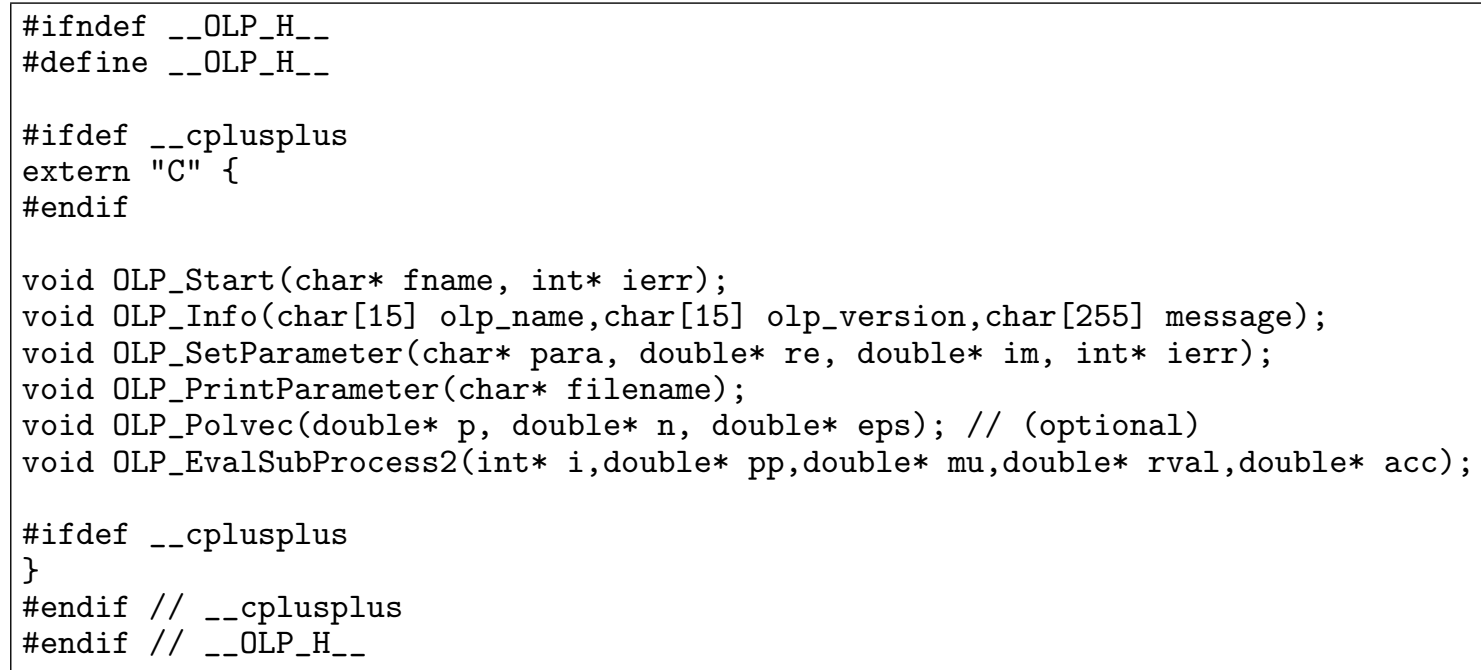

\section{References}

[1] T. Binoth, F. Boudjema, G. Dissertori, A. Lazopoulos, A. Denner, et al., A proposal for a standard interface between Monte Carlo tools and one-loop programs, Comput. Phys. Comm. 181 (2010) 1612-1622. 1001.1307.

[2] T. Gleisberg, F. Krauss, Automating dipole subtraction for QCD NLO calculations, Eur. Phys. J. C 53 (2008) 501-523. 0709.2881.

[3] T. Gleisberg, S. Höche, F. Krauss, M. Schönherr, S. Schumann, et al., Event generation with SHERPA 1.1, J. High Energy Phys. 0902 (2009) 007.0811 .4622$.

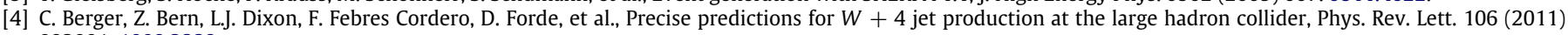
092001. 1009.2338.

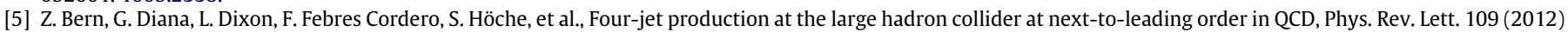
042001. 1112.3940.

[6] R. Frederix, S. Frixione, K. Melnikov, G. Zanderighi, NLO QCD corrections to five-jet production at LEP and the extraction of $\alpha_{s}\left(M_{Z}\right)$, J. High Energy Phys. 1011 (2010) 050. 1008.5313.

[7] V. Hirschi, R. Frederix, S. Frixione, M.V. Garzelli, F. Maltoni, et al., Automation of one-loop QCD corrections, J. High Energy Phys. 1105 (2011) 044. 1103.0621.

[8] G. Cullen, N. Greiner, G. Heinrich, G. Luisoni, P. Mastrolia, et al., Automated one-loop calculations with GoSam, Eur. Phys. J. C 72 (2012) 1889. 1111.2034.

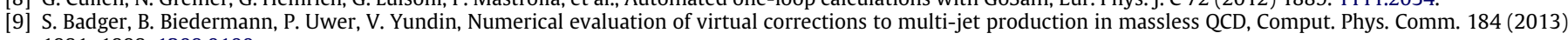
1981-1998. 1209.0100.

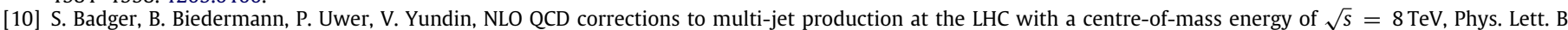
718 (2013) 965-978. 1209.0098.

[11] S. Alioli, S.-O. Moch, P. Uwer, Hadronic top-quark pair-production with one jet and parton showering, J. High Energy Phys. 1201 (2012) 137.1110 .5251$.

[12] E. Re, NLO corrections merged with parton showers for $Z+2$ jets production using the POWHEG method, J. High Energy Phys. 1210 (2012) 031 . 1204.5433.

[13] G. Cullen, N. Greiner, G. Heinrich, Susy-QCD corrections to neutralino pair production in association with a jet, Eur. Phys. J. C 73 (2013) 2388.1212 .5154$.

[14] G. Luisoni, P. Nason, C. Oleari, F. Tramontano, Merging $H W / H Z+0$ and 1 jet at NLO with no merging scale using the POWHEG BOX interfaced to GoSam, 1306.2542.

[15] Z. Bern, L. Dixon, F. Febres Cordero, S. Höche, H. Ita, et al. Next-to-leading order W + 5-jet production at the LHC, 1304.1253.

[16] S. Höche, J. Huang, G. Luisoni, M. Schönherr, J. Winter, Zero and one jet combined NLO analysis of the top quark forward-backward asymmetry, 1306.2703.

[17] G. Bevilacqua, M. Czakon, M. Garzelli, A. van Hameren, A. Kardos, et al., HELAC-NLO, Comput. Phys. Comm. 184 (2013) 986-997. 1110.1499.

[18] F. Cascioli, P. Maierhofer, S. Pozzorini, Scattering amplitudes with open loops, Phys. Rev. Lett. 108 (2012) 111601. 1111.5206.

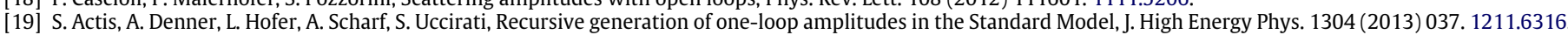

[20] M. Bahr, S. Gieseke, M. Gigg, D. Grellscheid, K. Hamilton, et al., Herwig++ physics and manual, Eur. Phys. J. C 58 (2008) 639-707. 0803.0883.

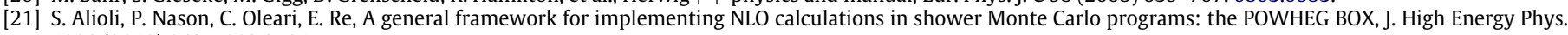
1006 (2010) 043. 1002.2581.

[22] J. Alwall, M. Herquet, F. Maltoni, O. Mattelaer, T. Stelzer, MadGraph 5: going Beyond, J. High Energy Phys. 1106 (2011) 128.1106 .0522$.

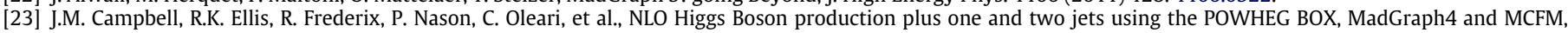
J. High Energy Phys. 1207 (2012) 092. 1202.5475. 


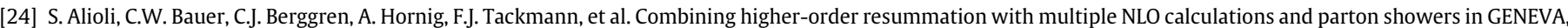
1211.7049.

[25] M. Ritzmann, D. Kosower, P. Skands, Antenna showers with hadronic initial states, Phys. Lett. B 718 (2013) 1345-1350. 1210.6345.

26] R. Frederix, T. Gehrmann, N. Greiner, Automation of the dipole subtraction method in MadGraph/MadEvent, J. High Energy Phys. 0809 (2008) 122. 0808.2128.

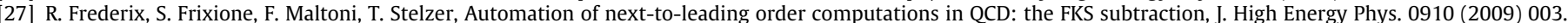
0908.4272.

[28] Particle Data Group Collaboration, J. Beringer, et al., Review of particle physics (RPP), Phys. Rev. D 86 (2012) 010001.

29] C. Degrande, C. Duhr, B. Fuks, D. Grellscheid, O. Mattelaer, et al., UFO-the universal feynrules output, Comput. Phys. Comm. 183 (2012) 1201-1214. 1108.2040.

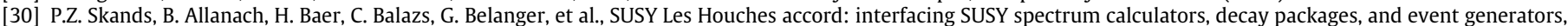
J. High Energy Phys. 0407 (2004) 036. hep-ph/0311123.

[31] T. Hahn, SUSY Les Houches accord 2 I/O made easy, Comput. Phys. Comm. 180 (2009) 1681-1693. hep-ph/0605049.

[32] B. Allanach, C. Balazs, G. Belanger, M. Bernhardt, F. Boudjema, et al., SUSY les houches accord 2, Comput. Phys. Comm. 180 (2009) 8-25. 0801.0045.

[33] S. Höche, F. Krauss, M. Schönherr, F. Siegert, QCD matrix elements + parton showers: the NLO case, J. High Energy Phys. 1304 (2013) 027. 1207.5030.

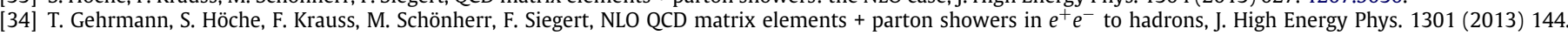
1207.5031

[35] R. Frederix, S. Frixione, Merging meets matching in MC@NLO, J. High Energy Phys. 1212 (2012) 061. 1209.6215

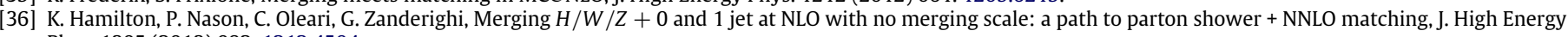
Phys. 1305 (2013) 082. 1212.4504.

37] S. Plätzer, Controlling inclusive cross sections in parton shower + matrix element merging 1211.5467.

[38] L. Lonnblad, S. Prestel, Merging multi-leg NLO matrix elements with parton showers, J. High Energy Phys. 1303 (2013) 166. 1211.7278.

[39] K. Hamilton, P. Nason, G. Zanderighi, MINLO: multi-scale improved NLO, J. High Energy Phys. 1210 (2012) 155. 1206.3572.

[40] S. Catani, M. Seymour, A General algorithm for calculating jet cross-sections in NLO QCD, Nuclear Phys. B 485 (1997) 291-419. hep-ph/9605323. 